\title{
THE ENRICHMENT OF THE INTRACLUSTER MEDIUM
}

Galactic Winds, Dwarf Galaxies, and Bimodal Star Formation in Ellipticals

\author{
B.K. GIBSON \\ Mt. Stromlo $\mathcal{G}$ Siding Spring Observatories \\ Australian National Univ., Weston Creek, Australia ACT 2611 \\ AND \\ F. MATTEUCCI \\ Univ. of Trieste \\ Dept. of Astronomy, Via G.B. Tiepolo 11, Trieste, Italy 34131
}

\section{Overview}

Recent observational evidence for steep dwarf galaxy luminosity functions in several rich clusters has led to speculation that their precursors, via supernovae (SNe)-driven galactic winds, may be the source of the majority of gas and metals inferred from intracluster medium (ICM) $\mathrm{x}$-ray observations (Trentham 1994). Utilising a fully self-consistent photo-chemical evolution package (Gibson 1995), and insisting that the post-galactic wind dwarfs obey the observed colour-luminosity-metallicity relations, we demonstrate that the bulk of the ICM gas does not originate within their precursors (Gibson \& Matteucci 1995).

In a parallel study, we consider the present-day photo-chemical properties of elliptical galaxies, adopting the bimodal star formation scenario of Elbaz, Arnaud \& Vangioni-Flam (1995). Based upon chemical evolution arguments alone, this scenario has been invoked by them to explain the observed metal mass, and their abundance ratios, in the ICM of galaxy clusters. Our fully self-consistent photo-chemical evolution analysis of their model highlights its failings: their predicted V-K colours are $>1$ mag too red; their luminosity-weighted metallicities $>0.7$ dex too high; and their predicted metallicity dispersion is virtually non-existent $(<0.2 \mathrm{dex}$, versus the $>3$ dex implied observationally) (Gibson 1995).

\section{References}

Elbaz, D., Arnaud, M., Vangioni-Flam, E., 1995, A\&A, in press (and these proceedings) Gibson, B.K., 1995, MNRAS, in press

Gibson, B.K., Matteucci, F., 1995, MNRAS, submitted

Trentham, N., 1994, Nature, 372, 157 